\title{
Coherent Ptychographic Imaging Microscope With 17.5nm Spatial Resolution Employing 13.5nm High Harmonic Light
}

Michael Tanksalvala ${ }^{1}$, Dennis F. Gardner ${ }^{1}$, Giulia F. Mancini ${ }^{1}$, Elisabeth R. Shanblatt ${ }^{1}$, Xiaoshi Zhang ${ }^{2}$, Benjamin R. Galloway ${ }^{1}$, Christina R. Porter ${ }^{1}$, Robert Karl Jr. ${ }^{1}$, Charles Bevis ${ }^{1}$, Margaret M. Murnane ${ }^{1,2}$, Henry Kapteyn $^{1,2}$ and Daniel E. Adams ${ }^{1}$.

1. JILA, University of Colorado, Boulder USA.

2. Kapteyn-Murnane Laboratories, Boulder USA.

High-resolution imaging is an essential tool for understanding nanoscale systems. In particular, tabletop extreme ultraviolet (EUV) coherent diffractive imaging (CDI) techniques based on high harmonic generation (HHG) are ideal for investigating complex nanostructured systems, including their static and dynamic electronic, phononic and magnetic properties. Tabletop EUV CDI combines elemental and chemical selectivity with nanometer spatial resolution, with pulse durations in the femtosecond (fs)-toattosecond (as) range [1-5]. In this work, we use ptychographic CDI [6-7] with high-spatial-coherence $13.5 \mathrm{~nm}$ tabletop HHG to obtain $17.5 \mathrm{~nm}$ spatial resolution images of a zone plate. This is the highest demonstrated resolution for a full-field tabletop microscope using any light source.

In CDI, also known as lensless imaging, a coherent beam illuminates an object and the diffracted light is directly recorded on a charge-coupled device (CCD) far from the sample. The phase profile of the diffraction pattern, lost by the detector, is recovered computationally, and the object is reconstructed by numerically back-propagating this field [8]. In a newly-developed form of CDI called ptychography, many diffraction patterns are recorded as an illuminating beam is scanned across a sample. These patterns are combined with a phase retrieval algorithm to reconstruct the complex profiles of the object and probe beam. Ptychography provides excellent image fidelity compared to other techniques such as scanning electron microscope (SEM) imaging $[1,3,6]$, requires no contact with the sample, has a working distance of centimeters and does not suffer from adverse effects such as surface charging.

We used an actively stabilized $13.5 \mathrm{~nm}$ HHG source (KM Labs XUUS 4.0) driven by a $20 \mathrm{fs}, 2 \mathrm{~mJ}, 3 \mathrm{kHz}$, Ti:Sapphire laser centered at 785nm (KM Labs Dragon), and generated a flux which was 10 times higher than was previously possible using the same driving laser [2]. The HHG light was produced in a $150 \mu \mathrm{m}$ diameter waveguide filled with 500 Torr of He. Differential pumping helped maintain high vacuum before and after the waveguide. After the waveguide, the residual IR light was attenuated using a pair of $\mathrm{ZrO}_{2}$ coated Si mirrors placed near Brewster's angle, together with a single $600 \mathrm{~nm}$ thick $\mathrm{Zr}$ foil filter. A single harmonic was then selected using a pair of Si/Mo multilayer mirrors.

The imaging setup is depicted in Fig. 1a. A flat mirror is followed by a curved mirror (radius of curvature $100 \mathrm{~mm}$ ), which focuses the beam onto the sample. The angle of incidence on the curved mirror is approximately $2.5^{\circ}$, resulting in a $2.7 \mu \mathrm{m} 1 / \mathrm{e}^{2}$ diameter at the circle of least confusion. Diffraction from a zone plate test sample was collected by a CCD detector (Andor iKon, 2048x2048 pixels, $13.5 \mu \mathrm{m}$ pitch) placed $22.6 \mathrm{~mm}$ from the sample. This geometry provided a numerical aperture (NA) of 0.4 , corresponding to a half-pitch resolution of $17.5 \mathrm{~nm}$. The beam was scanned in an $11 \mathrm{x} 11$ grid with $\approx 0.8 \mu \mathrm{m}$ steps, with a total exposure time (121 diffraction patterns, two accumulations each) of 17 minutes $\left(16 \mathrm{~s} / \mu \mathrm{m}^{2}\right)$. We used the ePIE algorithm with position correction for reconstruction [6-7]. 
An SEM image of the zone plate is shown for comparison with the amplitude retrieved by ptychography, as shown respectively in Fig. 1(b, c). Zoomed-frames of the SEM and CDI amplitude and phase images (Fig. 2(b, c, d) respectively) show the fine details of the outer rings of the zone plate. The retrieved probe (wavelength $13.5 \mathrm{~nm}$ ) is displayed in Fig. 2a. The reconstructions in Fig. 2(c, d) show good agreement with the SEM image of the same region.

We demonstrated a novel, full-field, transmission-mode, EUV HHG microscope for ptychographic imaging on a tabletop, with $13.5 \mathrm{~nm}$ illumination. This microscope enabled us to non-destructively image a zone plate with $<20 \mathrm{~nm}$ spatial resolution. In the future, it can be combined with pump-probe techniques to image energy, charge and spin transport with nanometer spatial and femtosecond temporal resolution, to uncover the mechanisms underlying function in nanostructured materials and interfaces.

\section{References:}

[1] B. Zhang et al, Ultramicroscopy 158 (2015), p. 98-104.

[2] M. Seaberg et al, Opt. Express 19 (2011), p. 22470-22479.

[3] M. Seaberg et al, Optica 1 (2014), p. 39-44.

[4] J. Miao et al, Science 348 (2015), p. 530-535.

[5] E. Turgut et al, Physical Review Letters 110 (2013), p. 197201.

[6] A. Maiden and J. Rodenburg, Ultramicroscopy 109 (2009), p. 1256-1262.

[7] F. Zhang et al, Opt. Express 21 (2013), p. 13592-13606.

[8] J. Fienup, Opt. Lett. 3 (1978), p. 27-29.

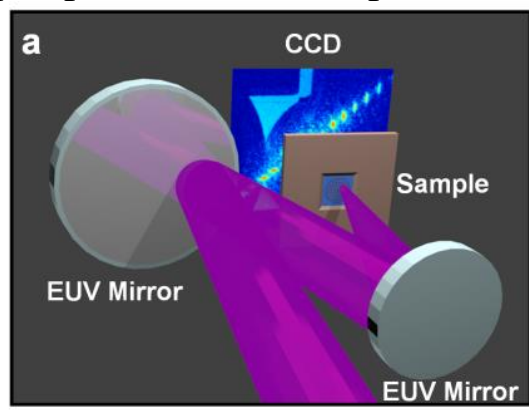

Experimental setup

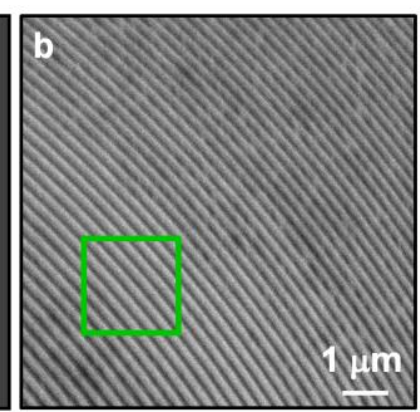

SEM

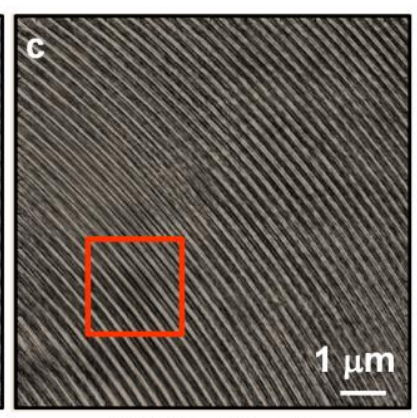

CDI Amplitude

Figure 1. (a) Schematic of the coherent EUV imaging geometry. A pair of multilayer mirrors select and focus the $13.5 \mathrm{~nm}$ harmonic onto the zone plate sample. (b) A scanning electron microcopy (SEM) image and (c) our EUV ptychography amplitude reconstruction of the same region of the zone plate.

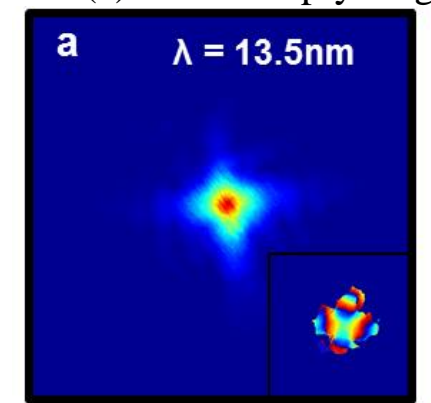

Probe

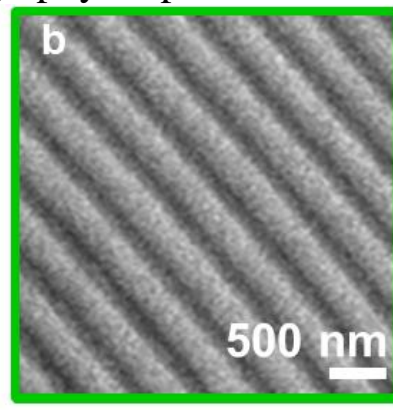

SEM

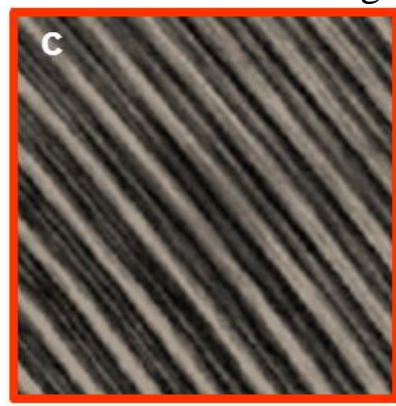

CDI Amplitude

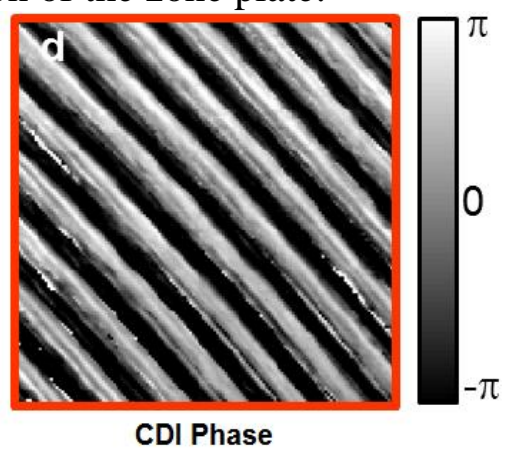

CDI Phase

Figure 2. Details of the zone plate sample from the highlighted regions in Fig. 1b and Fig. 1c. (a) The retrieved illumination on the sample with the inset showing the phase. (b) The SEM image and the (c) phase and (d) amplitude of the EUV CDI image. The scale bar in (b) is shared with (c) and (d). 